\title{
Cross-Whisker Adaptation of Neurons in the Rat Barrel Cortex
}

\author{
Yonatan Katz, ${ }^{\star}$ Jaime E. Heiss, ${ }^{*}$ and Ilan Lampl \\ Department of Neurobiology, The Weizmann Institute of Science, 76100 Rehovot, Israel
}

\begin{abstract}
Neurons in the barrel cortex and the thalamus respond preferentially to stimulation of one whisker (the principal whisker) and weakly to several adjacent whiskers. Cortical neurons, unlike thalamic cells, gradually adapt to repeated whisker stimulations. Whether cortical adaptation is specific to the stimulated whisker is not known. The aim of this intracellular study was to determine whether the response of a cortical cell to stimulation of an adjacent whisker would be affected by previous adaptation induced by stimulation of the principal whisker and vice versa. Using a high-frequency stimulation that causes substantial adaptation in the cortex and much less adaptation in the thalamus, we show that cortical adaptation evoked by a train of stimuli applied to one whisker does not affect the synaptic response to subsequent stimulation of a neighboring whisker. Our data indicate that intrinsic mechanisms are not involved in cortical adaptation. Thalamic recordings obtained under the same conditions demonstrated that an adjacent whisker response was not generated in the thalamus, indicating that the observed whisker-specific adaptation results from diverging thalamic inputs or from cortical integration.
\end{abstract}

Key words: intracellular recording; in vivo; layer 4; receptive field; intracortical connections; VPM; vibrissa

\section{Introduction}

Sensory adaptation is common to different sensory modalities. Changes in sensitivity after adaptation might allow the neuronal circuit to respond optimally to a large range of stimulation despite the limited dynamic range of neuronal firing (Adorjan et al., 1999; Muller et al., 1999). Studies of the visual (Movshon and Lennie, 1979; Saul and Cynader, 1989; Dragoi et al., 2000), auditory (Condon and Weinberger, 1991; Malone et al., 2002; Ulanovsky et al., 2003), and somatosensory (Derdikman et al., 2006) cortices suggest that adaptation is usually stimulus specific.

Sensory adaptation in the barrel cortex is frequency dependent. During exploration, rats sweep their whiskers at $5-15 \mathrm{~Hz}$, and, during foveal whisking, frequency can increase to $15-25 \mathrm{~Hz}$ (Berg and Kleinfeld, 2003). Repetitive stimulation above $10 \mathrm{~Hz}$ strongly suppresses the response of cells in the posterior medial nucleus (POm) of the thalamus (Ahissar et al., 2000; Sosnik et al., 2001). Cells in the ventral posterior medial nucleus (VPM) of the thalamus, which exhibit shorter response latency compared with POm cells, are highly responsive at the entire frequency range of whisking. Cortical cells, however, undergo profound gradual adaptation (Ahissar et al., 2000, 2001; Sosnik et al., 2001; Khatri et al., 2004; Gabernet et al., 2005), which is thought to reflect short-

Received Sept. 17, 2006; revised Nov. 15, 2006; accepted Nov. 17, 2006.

This work was supported by The Israel Science Foundation Grant 1037/03, Henry S. and Anne Reich Research Fund for Mental Health, and the Asher and Jeanette Alhadeff Research Award. I.L. is an incumbent of the Carl and Frances Korn Career Development Chair in the Life Sciences. We thank Ehud Ahissar for his critical comments on our paper. We thank Valerie Mazig for assistance in histology and all of the members of Lampl laboratory for their helpful contribution to this work.

*Y.K. and J.E.H. contributed equally to this work.

Correspondence should be addressed to Dr. llan Lampl, Department of Neurobiology, The Weizmann Institute of Science, 76100 Rehovot, Israel. E-mail: ilan.lampl@weizmann.ac.il.

DOI:10.1523/JNEUROSCI.4056-06.2006

Copyright $\odot 2006$ Society for Neuroscience ～0270-6474/06/2613363-10\$15.00/0 term synaptic depression of thalamocortical synapses (Gil et al., 1999; Castro-Alamancos, 2002; Chung et al., 2002; Gabernet et al., 2005; Bruno and Sakmann, 2006). However, because cortical cells respond to several whiskers, the whisker specificity of adaptation in the barrel cortex remains unclear. In theory because cortical cells respond to several whiskers, repetitive stimulation of one whisker might cause reduced responsiveness to subsequent stimulation of neighboring whiskers. Whether or not this will occur depends not only on cellular and synaptic mechanisms of adaptation but also on the synaptic organization of inputs from different whiskers. In layer 4 of the barrel cortex (Woolsey and Van der Loos, 1970), a stronger response is evoked by stimulation of the principal whisker (PW) and a weaker response by stimulation of an adjacent whisker (AW) (Simons, 1985). A multiwhisker receptive field characterizes cells above and below layer 4 (Ghazanfar and Nicolelis, 1999; Brecht et al., 2003; Manns et al., 2004). Feedforward thalamic inputs are evidently the main source for the PW response in cortical neurons. Regarding the origin of the AW response, two main mechanisms have been suggested. (1) Multiwhisker receptive field can be found in the VPM; thus, multiwhisker receptive fields of cortical neurons may already be synthesized subcortically (Simons and Carvell, 1989; Goldreich et al., 1999; Kwegyir-Afful et al., 2005). (2) The AW response might be generated by intracortical interactions (Armstrong-James and Callahan, 1991; Brecht and Sakmann, 2002b; Fox et al., 2003). If multiwhisker receptive field is generated subcortically, adaptation of different whiskers would not be expected to be independent.

We show that, despite profound adaptation induced by repetitive stimulation of one whisker, the cortical synaptic response to subsequent stimulation of a neighboring whisker is barely affected. Our findings point to possible mechanisms of sensory 
adaptation and may shed light on the functional connectivity of the somatosensory system.

\section{Materials and Methods}

Animal preparation and recording. Forty-eight young adult Wistar rats (4-8 weeks old) were initially anesthetized with ketamine $(100 \mathrm{mg} / \mathrm{kg}$, i.p.; Ketaset; Fort Dodge Animal Health, Fort Dodge, IA) and acepromazine maleate ( $1 \mathrm{mg} / \mathrm{kg}$, i.p; PromAce; Fort Dodge Animal Health). After tracheotomy, a short $(1.5 \mathrm{~cm})$ metal cannula [outer diameter (o.d.), 2 $\mathrm{mm}$; inner diameter (i.d.), $1.5 \mathrm{~mm}$ ] was inserted into the trachea. The rat was placed in a standard stereotaxic device. Body temperature was kept at $37.0 \pm 0.1^{\circ} \mathrm{C}$ using a heating blanket and a rectal thermometer (TC-1000; CWE, Ardmore, PA). Anesthesia was maintained, using a mixture of halothane $(0.5-1.5 \%)$ and air, by means of artificial respiration at a rate of 100-115 breaths/min while monitoring the levels of end-tidal $\mathrm{CO}_{2}$ and heart rate. Anesthesia was monitored by heart rate (250-450 beats/ min), eyelid reflex, pinch withdrawal, and vibrissal movements. Halothane concentration was set slightly above the level at which the first clear signs of vibrissal movements were observed, while the eyelid reflex was still maintained. In seven control animals, we carefully analyzed these parameters, as well as EEG recordings obtained by means of two wires inserted under the skull at a distance of $9 \mathrm{~mm}$ anterocaudally. Based on these measurements, we assessed the anesthesia level used in our recordings to be between stages III-2 and III-3 (Friedberg et al., 1999).

A craniotomy, $1 \mathrm{~mm}$ in diameter, was performed above the barrel cortex (centered at $2.5 \mathrm{~mm}$ posterior and $5.2 \mathrm{~mm}$ lateral to the bregma), and the dura mater was carefully removed. All surgical and experimental procedures were in accordance with the regulations of The Weizmann Institute Animal Care and Use Committee.

Recording techniques. "Blind” sharp electrode (Lampl et al., 1999) and patch recording (Chung and Ferster, 1998; Priebe and Ferster, 2005) techniques were used in this study to record the membrane potential of cortical cells. Sharp recordings were obtained using borosilicate micropipettes (o.d., $1.5 \mathrm{~mm}$; i.d., $0.86 \mathrm{~mm}$; Sutter Instruments, Novato, CA), pulled with a P-97 micropipette puller (Sutter Instruments), and filled with $2 \mathrm{M} \mathrm{K}$-acetate with a resistance of $60-100 \mathrm{M} \Omega$. Patch electrodes were pulled using the same glass and puller and were filled with an intracellular solution containing the following (in $\mathrm{mm}$ ): $136 \mathrm{~K}$-gluconate, $10 \mathrm{KCl}, 5$ $\mathrm{NaCl}, 10$ HEPES, 1 MgATP, $0.3 \mathrm{NaGTP}$, and 10 phosphocreatine (310 mOsm). Patch recording electrodes contained biocytin $(0.4 \%)$ for histological identification of the recorded cells and QX-314 [N-(2,6dimethylphenylcarbamoylmethyl)triethylammonium bromide) acetamine] (2 mM) to prevent spikes. Patch electrode resistance was 5-8 $\mathrm{M} \Omega$, and series resistance during whole-cell recording was 30-130 M $\Omega$. QX314 also blocks persistent sodium channels and some potassium channels; therefore, it may affect the shape of the evoked PSP. At low internal concentration $(\sim 1 \mathrm{mM})$, QX-314 blocks fast and persistent sodium currents (Connors and Prince, 1982). At $5 \mathrm{~mm}$ concentration, it blocks the hyperpolarization-activated inward current $I_{\mathrm{h}}$ (Perkins and Wong, 1995). Higher concentration of QX-314 (50 mM) was found to block the $\mathrm{GABA}_{\mathrm{B}}$ synaptic component (Nathan et al., 1990). Nevertheless, the shape of the synaptic response was independent of the recording pipettes used in this study (see Results), suggesting that QX-314, used only in the patch pipettes, did not have a significant effect on the subthreshold response to whisker stimulation. To protect the brain from drying, we covered the craniotomy with a few drops of warm agar $3.5 \%$ in normal saline) after the electrode was positioned a few micrometers inside the cortex. Electrodes were inserted lateromedially using a motorized manipulator (MX7600; Siskiyou, Grants Pass, OR) at $60^{\circ}$ relative to the horizontal plane (approximately perpendicular to the cortical surface). Whole-cell patch recording was obtained under current-clamp mode while applying an initial positive pressure of $200 \mathrm{mbar}$, which was reduced inside the brain to 40-80 mbar. When an increase in resistance was observed, we removed the pressure and applied a small negative pressure to create a high-resistance seal $(>1 G \Omega)$. We then briefly applied stronger suction to create a whole-cell recording configuration. Signals were amplified using Axoclamp-2B (Molecular Devices, Palo Alto, CA) and low passed at $3 \mathrm{kHz}$ before being digitized at $15 \mathrm{kHz}$. For recordings from the VPM, we performed a craniotomy ( $1 \mathrm{~mm}$ in diam- eter; $3.0 \mathrm{~mm}$ posterior and $2.7-3.0 \mathrm{~mm}$ lateral to the bregma), and the dura was removed. Sharp electrodes were advanced vertically, and recordings were obtained at depths of $4.5-5.1 \mathrm{~mm}$. To verify that the recordings were obtained from the VPM, in three experiments, we made lesions ( $1 \mathrm{M} \Omega$ impedance, $0.5 \mathrm{~mA}$ for $1 \mathrm{~s}, 10$ times) in the recording coordinates.

Whisker stimulation. Whiskers were mechanically stimulated using a piezoelectric actuator (T220-H4-203Y; Piezo Systems, Cambridge, MA). The tip of the whisker was trimmed $10-15 \mathrm{~mm}$ from the base. To secure the whisker to the stimulator, it was inserted into a $2 \mathrm{~mm}$ plastic cone (made from a Microloader; Eppendorf, Hamburg, Germany) that was tightly inserted and glued into a short $(2 \mathrm{~cm})$ metal pipette (made from 20 gauge needles) attached to the piezoelectric wafer driven by a home-made piezo-controller. The displacement, rise time, and velocity (measured from 10 to $90 \%$ of the maximal amplitude) of the piezoelectric actuator (at the tip of the metal pipette) were calibrated off-line using a noncontact optical displacement measuring system (optoNCDT 1605; Micro-Epsilon, Ortenburg, Germany) with a time resolution of $0.1 \mathrm{~ms}$ and displacement resolution of $0.1 \mu \mathrm{m}$. Whisker deflection was obtained by delivering fast-rising voltage commands to evoke fast deflection $(30-40 \mathrm{~mm} / \mathrm{s})$ followed by a $20 \mathrm{~ms}$ ramp-down signal that returned the whisker to the baseline position. Examples of command signals are shown in Figure 6. Direction was always caudorostral. The intrinsic resonance of the piezoelectric actuators was $400 \mathrm{~Hz}$, which could potentially alter the response. To study the effect of this resonance on the neuronal response, we used a custom-made closed-loop control system based on a real-time program (PID Control Toolset; LabVIEW RT; National Instruments, Austin, TX) that was used in combination with the noncontact optical displacement system (supplemental Fig. S1, available at www. jneurosci.org as supplemental material). In control experiments in which we used this system to eliminate the resonance to almost zero, cortical responses and thalamic local field potential were nevertheless almost identical to the control responses (supplemental Fig. S2, available at www.jneurosci.org as supplemental material). Therefore, no additional measures were taken to filter this high-frequency mechanical artifact.

Receptive fields were mapped by probing different whiskers while averaging the response to 25 stimuli at a frequency of $0.5 \mathrm{~Hz}$. The two most responsive whiskers (the PW and one of the AWs) were selected on the basis of the amplitude of the averaged response (also see Results). To study cross-whisker adaptation, we used two stimulators attached to the two selected whiskers, and two types of trials were delivered. The first consisted of 20 stimuli (each stimulus voltage command shape was fastrising followed by slow ramp-down signal, as mentioned above, and delivered at $18 \mathrm{~Hz}$ ), which were applied to one whisker and followed immediately by a single stimulation of the neighboring whisker at the same interval ( $1 / 18 \mathrm{~s})$, shape, and amplitude. The second trial was identical to the first except that the two sets of stimulation were applied in the opposite order. The two trials were randomly repeated 20-70 times with an intertrial interval of $2.4 \mathrm{~s}$.

Data analysis. To quantify the strength of adaptation, we used frequency-domain analysis (also see Results). After delivery of a train of 20 stimuli at $18 \mathrm{~Hz}$, we computed the absolute value of the discrete Fourier transform of the average response using the Fast Fourier Transform algorithm (FFT). In addition, we computed the absolute value of the FFT of the expected response in the absence of adaptation by replicating the first cycle of the response 19 times to construct an artificial trace. These computations are equivalent to computing the FFT of the average of the 20 responses and the FFT of the first response in a train.

We used the term responsiveness index (RI) to denote the ratio of the magnitude of the Fourier component at the stimulation frequency of the average response to the corresponding value of the expected response. An RI value of 1 means that no adaptation was measured, whereas near-zero RI represents a lack of repetitive response at the stimulation frequency and thus denotes profound adaptation. Clearly, RI depends also on the number of stimuli. It can be shown, however, that the product of RI and the duration of the train is approximately equal to the time constant of an exponentially adapting response. Because we used the same frequency and equal numbers of stimuli in all measurements, we can compare RI values across different experiments. We chose frequency-domain analy- 

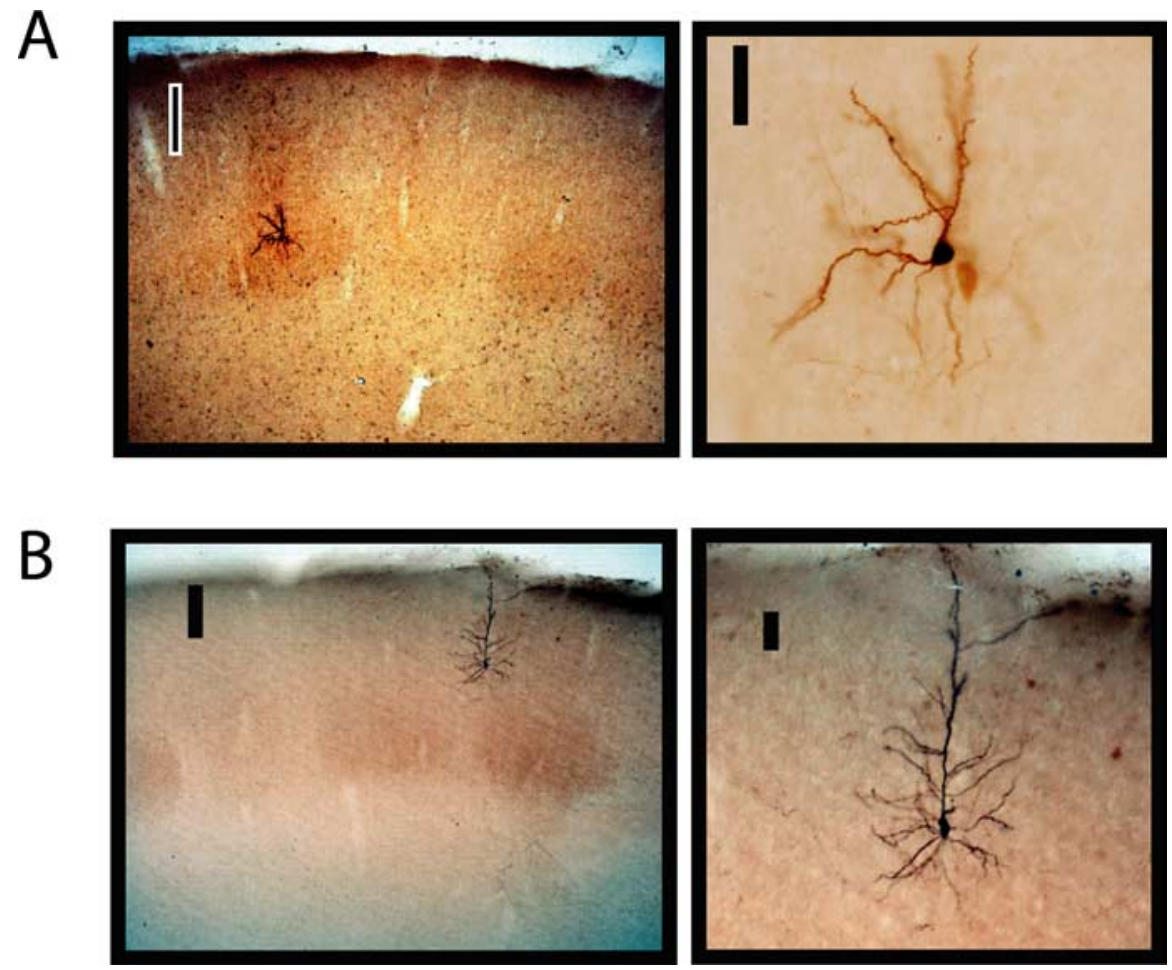

Figure 1. Barrel cortex neurons. A, Biocytin-filled spiny stellate neuron from layer 4. B, Pyramidal neuron from layer 2/3. Cells were recorded in different experiments, and sections were double stained for cytochrome c and biocytin. Scale bars: barrel cortex view, $200 \mu \mathrm{m}$; magnified cells, $50 \mu \mathrm{m}$.

sis rather than amplitude measurement of each response in a train, for two main reasons: (1) in many cells, non-monotonic decline in the responses was observed; and (2) any remaining noise of ongoing activity after averaging has a larger distorting effect on amplitude measurement in the time domain but less effect in the frequency domain. Student's $t$ test was used for statistical comparisons.

Histology. At the end of the experiment, the rats were overanesthetized and perfused transcardially with $2.5 \%$ paraformaldehyde, and their brains were removed and postfixed in the perfusion solution. After $24 \mathrm{~h}$, sections (100 $\mu \mathrm{m}$ thick) were either cut in a vibratome (Vibratome 1000; Vibratome, Redding, CA) or immersed in paraformaldehyde solution with additional $25 \%$ sucrose for cryoprotection for $24 \mathrm{~h}$ and then cut in a microtome (SM 2000R; Leica, Heidelberg, Germany). Sectioning was performed at an orientation of $45^{\circ}$ from the coronal plane toward the sagittal plane with the medial border being anterior, allowing us to view the barrels of the same arc in one section (Manns et al., 2004). As described in detail below, the recorded cells were visualized by avidinbiotin-peroxidase staining (Horikawa and Armstrong, 1988), and barrel visualization was enhanced by cytochrome oxidase (CO) staining (Land and Simons, 1985). By using both stains together, we were able to identify the position of the cell with respect to the barrels at layer 4 (Fig. 1).

Cellular staining. After each of the following steps, the sections were washed five times with PBS, each time for $10 \mathrm{~min}$. Unless otherwise indicated, incubations were performed at room temperature. Sections were submerged for $15 \mathrm{~min}$ in $3 \% \mathrm{H}_{2} \mathrm{O}_{2}$ in PBS solution. They were then submerged for $1 \mathrm{~h}$ in $2 \%$ Triton X-100 in PBS, which was then replaced (without washing) by avidin-biotin-peroxidase complex (Vectastain ABC kit; Vector Laboratories, Burlingame, CA) in 1\% Triton X-100 solution and left overnight at $5^{\circ} \mathrm{C}$. Finally, sections were placed in $5 \mathrm{ml}$ of PBS containing 0.07\% diaminobenzidine (one Sigma Fast DAB tablet; Sigma, St. Louis, MO) and $0.02 \%$ urea hydrogen peroxide (one tablet) for $0.2-24 \mathrm{~h}$, until the recorded cell was visible.

Barrel staining. Sections with the recorded cell and two adjacent sections on each side were washed twice, each time for $10 \mathrm{~min}$ in $0.1 \mathrm{M}$ phosphate buffer at $\mathrm{pH} 7.4$ and then incubated in oxygenated phosphate buffer solution containing $0.008 \%$ cytochrome $c, 0.02 \%$ catalase, and
$0.05 \% 3,3^{\prime}$-diaminobenzidine for $2-6 \mathrm{~h}$ at $37^{\circ} \mathrm{C}$. After appearance of the typical darkening in layer 4 , sections were washed four times in PBS and mounted on gelatin-covered microscope slides. Mounted sections were air dried, dehydrated in ethanol solutions and xylene, and embedded in Entellan (ProSciTech, Thuringowa, Queensland, Australia).

Layer classification. Histological depth of layer 4 was identified by the $\mathrm{CO}$-enriched band of the barrels found between $550 \pm 41$ and $850 \pm 69 \mu \mathrm{m}$ below the surface (mean $\pm \mathrm{SD}$; $n=15$ ). Because of tissue shrinkage and the nonperpendicular penetration angle, the manipulator readout of the recorded cells was on average 1.3-fold deeper than the depth of the soma established by histology. Examples of two cells, one recorded from layer 4 and another from layer 2/3, are shown in Figure 1.

Cells that were not reconstructed histologically were classified according to the following criteria. (1) Cells were classified as putative layer 4 barrel neurons if they were recorded in the range of the barrel band (between 550 and $850 \mu \mathrm{m})$ and if the latency to PW deflection (defined as the latency from stimulus onset to clear deviation of the averaged membrane potential from baseline) was $<7 \mathrm{~ms}$ (Wilent and Contreras, 2005). (2) If the histological depth of the recorded cell was more superficial than the upper range of barrels (550 $\mu \mathrm{m}$ histologically), the cell was considered as a layer $2 / 3$ cell. Only one cell in this category exhibited latency shorter than $7 \mathrm{~ms}$, yet it was classified as layer $2 / 3$ cell. (3) Cells located at the range of the barrel band with a latency longer than $7 \mathrm{~ms}$ were regarded as suspected septal cells (Moore and Nelson, 1998; Brecht and Sakmann, 2002b; Brecht et al., 2003; Higley and Contreras, 2003, 2005).

\section{Results}

\section{Recorded population}

Thalamic recordings

Intracellular recordings of 14 VPM cells were obtained using sharp electrodes at an average manipulator depth of $4760 \pm 200$ $\mu \mathrm{m}$ beneath the pial surface. The mean resting membrane potential of VPM cells was $68 \pm 15 \mathrm{mV}$, and the latency to whiskerevoked spike was $5.3 \pm 0.8 \mathrm{~ms}$. The receptive field was mapped in 11 cells, and the response to repetitive stimulation was recorded in six cells.

\section{Cortical recordings}

Intracellular recordings were obtained from 42 cells of the barrel cortex in anesthetized rats. Seven cells were recorded using sharp electrodes, and 35 were recorded using potassium-based patch electrodes filled with QX-314. The results obtained by the two recording techniques did not differ significantly, and therefore data from all cells were grouped together. For example, the width of the PW-evoked PSP at half-amplitude obtained using patch pipettes $(26 \pm 7 \mathrm{~ms})$ was not significantly different $(p=0.71)$ from that recorded using sharp electrodes $(27 \pm 7 \mathrm{~ms}$, mean \pm SD). We recorded 19 cells from layer $2 / 3$ and 19 from layer 4 (see Materials and Methods). The remaining four cells were thought to be layer 4 septal cells and were excluded from the study.

For each cell, the receptive field was mapped by means of caudorostral deflection. In layer $4,26 \%$ of the cells (5 of 19) responded to only one whisker. Because our goal in this adaptation study was to compare the PW response with the AW re- 
sponse, these five cells were excluded from the analysis. Layer $2 / 3$ cells responded to PW deflection with latency (mean \pm SD) of $8.9 \pm 1.5 \mathrm{~ms}$, whereas layer 4 barrel cells responded with latency of $6.6 \pm 0.6 \mathrm{~ms}$.

\section{Response of thalamic cells to}

repetitive stimulation

Previous studies have suggested that neurons of the VPM adapt much less than cortical neurons (Chung et al., 2002; Hartings et al., 2003; Khatri et al., 2004). We therefore first examined the response of intracellularly recorded thalamic cells to repetitive whisker stimulation at $18 \mathrm{~Hz}$. An example of a single-trial response from one cell to a train of stimuli is presented in Figure $2 \mathrm{~A}$. An overlay of 10 trials (randomly selected from 50 repeating stimuli) showed that the latency of firing for the first stimulus was shorter than for subsequent stimuli (illustrated for the 10th and 20th stimuli) and that jitter in the response did not increase during adaptation. A peristimulus time histogram (PSTH) constructed from this cell (Fig. 2C) together with the mean PSTH for the cell population at $18 \mathrm{~Hz}$ (Fig. $2 D)(n=6)$ indicated that, at 18 $\mathrm{Hz}$, thalamic cells attain a steady state very rapidly and thus respond almost equally from the second stimulus to the last. This finding is in line with other VPM adaptation studies (Hartings et al., 2003; Khatri et al., 2004). The receptive fields of VPM cells were mostly limited to one whisker (Fig. 2D,E); only 1 cell of 11 recorded cells fired when adjacent whiskers were stimulated. Moreover, in the absence of firing, a clear subthreshold response for adjacent-whisker stimulation could not be observed (data not shown), indicating that the small suprathreshold receptive fields were not biased by the recording technique. VPM cells, therefore, show almost no gradual adaptation during a train of repetitive stimulation. Under our experimental conditions, the receptive fields of thalamic cells were mostly limited to a single whisker, suggesting that inputs that are activated by stimulation of different whiskers enter the cortex independently.

\section{Cortical subthreshold response to repetitive stimulation of the PW and the AW}

To examine the subthreshold responses of layer 4 and layer $2 / 3$ neurons to repetitive stimulation of the PW and the AW, we first compared the averaged responses of a layer 4 cell to stimulation of different whiskers (Fig. $3 A$ ) and the individual responses to stimulation of the PW and best AW (Fig. $3 B$ ). Stimulation of the PW evoked stronger responses with shorter latencies and less jitter than stimulation of the best AW. In addition, this layer 4 cell showed much more adaptation during repetitive stimulation of the AW than during stimulation of the PW (Fig. 3C,D, black traces). To estimate the extent of adaptation, we replicated the first average response 19 times (Fig. 3C,D, gray traces). We then calculated the absolute value of the discrete Fourier transform to the overall average response (Fig. $3 E, F$, black curves) and to the surrogate replicated data (Fig. $3 E, F$, gray lines) and measured the ratios of their corresponding values at the selected stimulation frequency $(18 \mathrm{~Hz}$ ), defined as the responsiveness index (also see Materials and Methods). As expected from the averaged responses of the cell shown in Figure $3, A$ and $B$, the RI of the
A

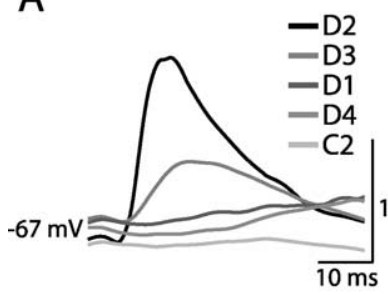

C

PSTH

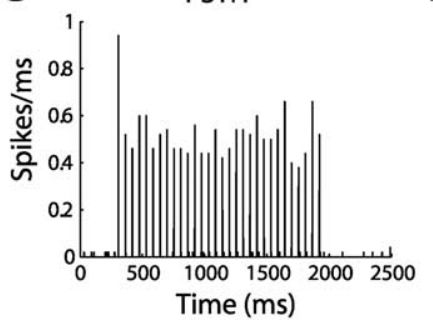

D

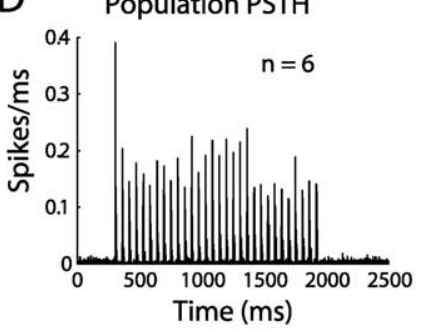

E Suprathreshold RF normalized

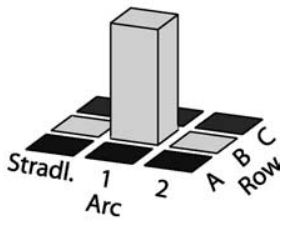

$\mathrm{F}$

Population RF normalized

$n=11$

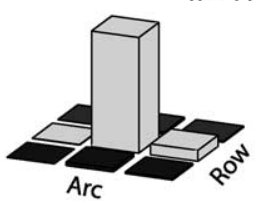

C PW

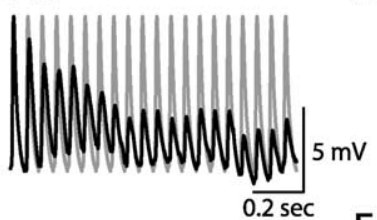

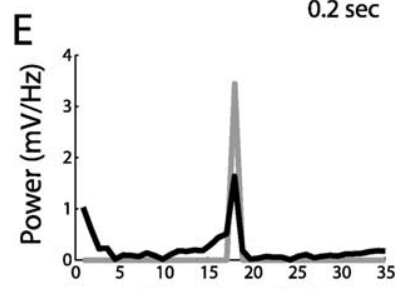

Frequency $(\mathrm{Hz})$
B

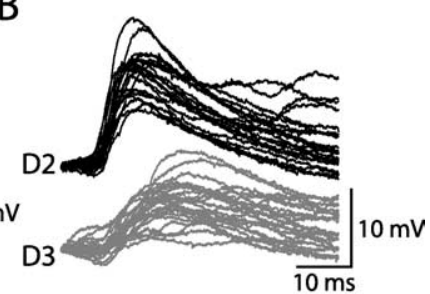

D AW
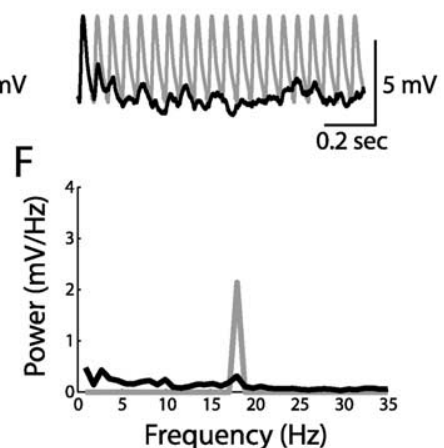

Figure 3. Membrane potential response of a layer 4 cell to repetitive stimulation of the PW and an AW. $A$, Average response of the cell to stimulation of five whiskers. $\boldsymbol{B}$, Individual responses to deflection of the PW (black) and the AW (gray). C, D, Average responses (shown in black) to repetitive stimulation (at $18 \mathrm{~Hz}$ ) of the PW $(\boldsymbol{C})$ and the AW $(\boldsymbol{D})$. The averaged responses are presented together with the expected response in the absence of adaptation (a trace made from the first response and its 19 replicas, shown in gray). $E$, $\boldsymbol{F}$, Absolute values of the discrete Fourier transform of the responses (black curve) together with the corresponding surrogate responses (gray curve) in $\boldsymbol{C}$ and $\boldsymbol{D}$. These curves were used to calculate the Rl as the ratio between the peak at $18 \mathrm{~Hz}$ and the corresponding value of the putative data.

response to PW stimulation was larger than the RI for AW stimulation (0.47 and 0.14 , respectively).

To measure the extent of adaptation in the population, we categorized each of the two best responsive whiskers according to 
A Latency sorted
by amplitude

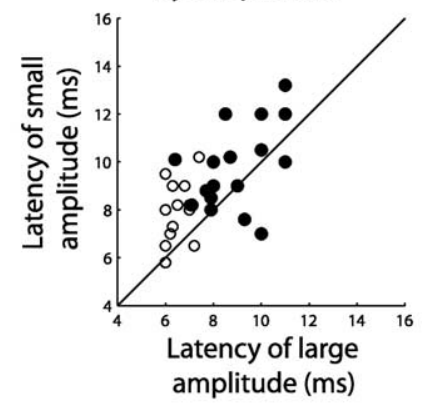

C Rl sorted by latency

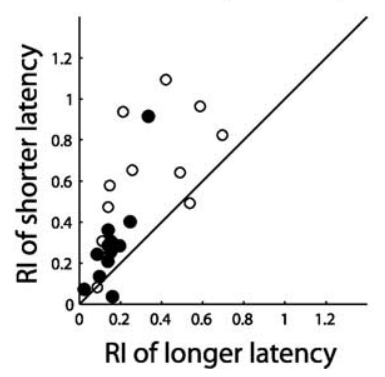

B

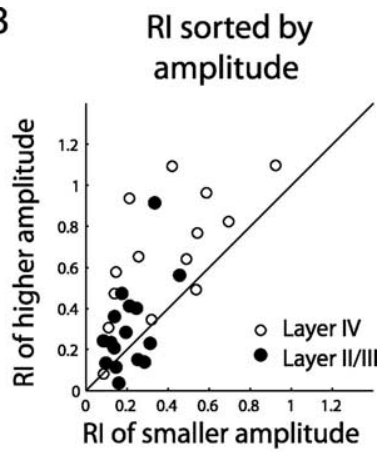

D

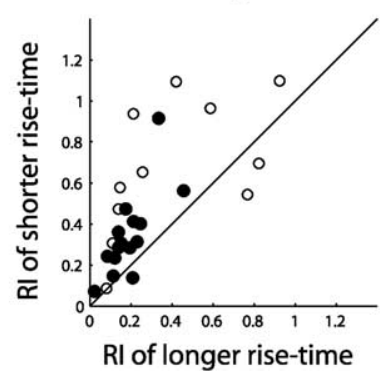

Figure 4. Barrel cortex neurons adapt less after repetitive stimulation of the PW than after stimulation of the AW. A, For each cell, the two whiskers that evoked the largest responses were selected. The latency of the smaller of the two responses (suspected AW) was plotted against the latency of the larger response (suspected PW). Layer 4 cells show shorter latency for larger response, whereas for layer $2 / 3$ cells the correlation is less clear-cut. $\boldsymbol{B}-\boldsymbol{D}$, Comparison of the extent of adaptation (inversely related to $\mathrm{RI}$ ) in terms of three different criteria for selection of the PW versus the AW: amplitude $(\boldsymbol{B})$, latency $(\boldsymbol{C})$, and rise time (D). The RI (see Materials and Methods) for the suspected AW is plotted in the abscissa and for the suspected PW in the ordinate. (ells of layer 4 are marked by open circles. Several cells ( 3 in $\boldsymbol{B}, 10$ in $\boldsymbol{C}$, and 7 in $\boldsymbol{D}$ ) were removed from the plots when criteria values for the two whiskers were barely indistinguishable (i.e., differing by $<10 \%$ ).

certain response parameters. The PW is identified by its higher response amplitude, shorter latency, and more rapid rise time relative to AW responses (Moore and Nelson, 1998; Brecht and Sakmann, 2002b; Brecht et al., 2003; Higley and Contreras, 2003, 2005). In line with these observed characteristics, for most cells of layer 4, the whisker that evoked a larger amplitude (defined as the peak voltage response minus baseline voltage at time of stimulation) also demonstrated a shorter latency (Fig. $4 \mathrm{~A}$, open circles) ( $p=0.0025$, paired $t$ test). In cells of layer $2 / 3$, however, this tendency was less clear (Fig. $4 A$, filled circles) but was still significant $(p=0.035)$. In most neurons, adaptation was less induced by repetitive stimulation of the whisker that evoked a response of larger amplitude, shorter latency, or faster rise time than that induced by stimulation of the other selected whisker. This tendency is shown in Figure $4 B-D$, in which the RI for the whisker that evoked the higher amplitude $(B)$, shorter latency $(C)$, and faster rise time $(D)$ is plotted against the RI measured for the other selected whisker. Note, however, that the amplitude of the response of layer $2 / 3$ cells was less correlated with the RI of the two selected whiskers when compared with latency and rise time. Across all cells, the mean RI for PW stimulation (determined by the latency criteria) was $0.41 \pm 0.29$ (mean $\pm \mathrm{SD} ; n=26)$ and was significantly larger ( $p=0.0002$, paired $t$ test) than the RI for AW stimulation $(0.22 \pm 0.15)$. Thus, the extent of adaptation, which we show is smaller for the whisker that evokes shorter latency, is a strong indicator for PW versus AW.

Adaptation varied substantially across the population (the RI

A

B

First stimulus

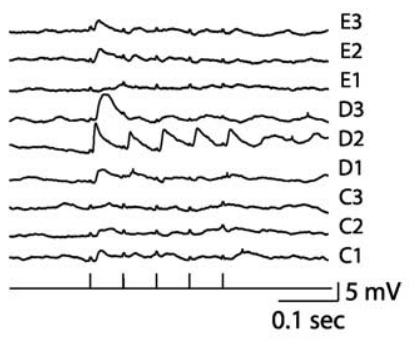

C

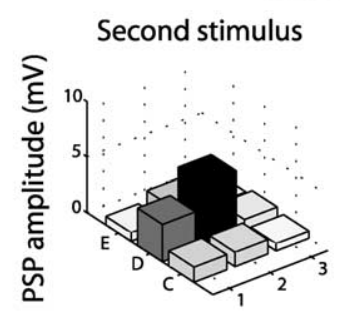

E

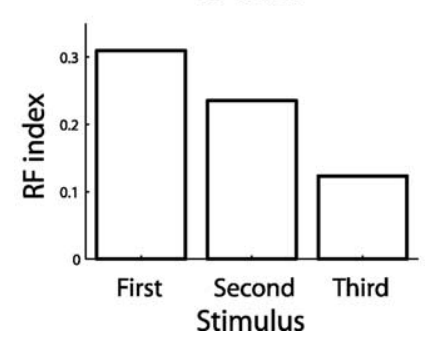

F Population RF index

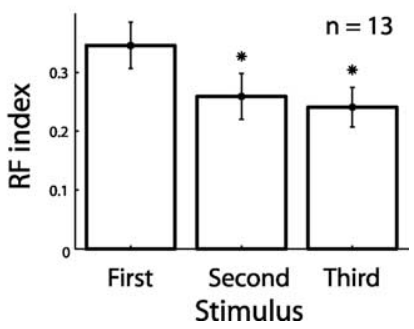

Figure 5. Subthreshold receptive field shrinks during adaptation. $A$, Average response of a layer 4 cell to repetitive stimulation at $18 \mathrm{~Hz}$ of nine different whiskers. Note that the response to the PW (D2) shows less adaptation than that of the other whiskers. $\boldsymbol{B}-\boldsymbol{D}$, Diagrams of the receptive field of the same cell $(\boldsymbol{A})$ constructed from the peak amplitude (measured from the maximal voltage during the first $40 \mathrm{~ms}$ after each stimulation minus the voltage at the time of stimulation) of the first $(\boldsymbol{B})$, second $(\boldsymbol{C})$, and third (D) responses to a train of stimuli. $\boldsymbol{E}$, Receptive field width measured as the mean ratio of the AW response to the PW response) of this cell was reduced during adaptation. $\boldsymbol{F}$, Average receptive field width of 13 cells shows significant reduction for the second and third responses to a train of stimuli. ${ }^{*} p<0.05$.

for PW stimulation ranged from $\sim 0.1$ to $\sim 1.1$ ). Others have shown that layer 4 neurons adapt less than neurons of layer $2 / 3$ (Ahissar et al., 2001; Ego-Stengel et al., 2005). Based on the RI of the PW response only, we found here that layer 4 cells (Fig. $4 B-D$, open circles) showed significantly less adaptation $(p=0.0053$, unpaired $t$ test) than cells above this layer (mean \pm SD RI values of layer 4 cells and layer $2 / 3$ cells were $0.65 \pm 0.25$ and $0.31 \pm$ 0.20 , respectively).

The large difference in adaptation during PW versus AW stimulation suggests that, during repetitive stimulation, the ratio between the response to stimulation of the PW and of other whiskers increases. In other words, the size of the receptive field shrinks during adaptation. We demonstrated this effect of different whiskers by mapping the receptive field using five deflections at $18 \mathrm{~Hz}$ (Fig. 5A). Each of the five stimuli evoked a clearly detectable subthreshold response only when the PW (whisker D2) was stimulated, whereas second and subsequent stimuli evoked much smaller responses to the stimulation of AWs. Plotting of the subthreshold receptive field for each of the first three stimuli disclosed a clear reduction in receptive field size (Fig. $5 B-D$ ). Receptive field width was quantified by calculating the ratio between the average surround whisker response and the PW response (Gabernet et al., 2005). Figure $5 E$ shows the receptive field 
width of each of the first three responses for a single cell (shown in Fig. $5 A-D$ ), and Figure $5 F$ demonstrates significant reduction in the width of the subthreshold receptive field during adaptation in a population of layer 4 cells.

\section{Cross-whisker adaptation}

Next we examined the effect of repetitive stimulation of a whisker on the response of a cell to subsequent stimulation of a neighboring whisker. We refer to this effect as "cross-whisker adaptation." Our results described above suggest that, under our experimental conditions, thalamic cells respond mostly to one whisker, whereas cortical cells respond to several whiskers. Thus, these findings suggest that sensory inputs from multiple whiskers are integrated at the cortical level. However, because of the small sample size of the thalamic cell population, we could not rule out the possibility that AW responses of cortical cells, under our experimental conditions, were generated by thalamic cells that responded to multiple whiskers. To clarify this issue, we examined the specificity of adaptation in the cortex. We reasoned that, if thalamic cells convey inputs from each whisker separately and if adaptation occurs at thalamocortical synapses (Chung et al., 2002), then adaptation of layer 4 cells should be specific to the stimulated whisker. Alternatively, reduced response of one whisker after the cell adapted by repetitive deflection of another one would be expected if either VPM neurons convey inputs from the two whiskers or if cortical mechanisms are involved in adaptation, for example, if the adaptation is mediated by intrinsic mechanisms such as activity-dependent opening of ion channels (Sanchez-Vives et al., 2000).

Cross-whisker adaptation was studied here using stimulation of $18 \mathrm{~Hz}$, which is high enough to induce a pronounced gradual adaptation in the cortex but almost no adaptation in the thalamus from the second response onward (Figs. 2-5) (Khatri et al., 2004). A train of 20 deflections of the AW, at a frequency of $18 \mathrm{~Hz}$, was followed by a single test deflection of the PW (Fig. 6A). Reciprocal testing of the AW was performed after repetitive stimulation of the PW (Fig. $6 \mathrm{~B}$ ). The pattern of command stimulation is depicted below the averaged membrane potential traces in Figure $6, A$ and $B$, in which the test stimulation is marked by an arrow. Despite profound adaptation of the response to stimulation of the AW, the test PW response was very similar to its corresponding control response measured from the averaged response to the first stimulus in a train of PW stimuli. The similarity of the PW test response to its control can be seen by comparing the last response in Figure $6 A$ with the first response in Figure $6 B$. Control and test responses are depicted in Figure 6C, together with the adapted response to the last deflection of the train (i.e., the 20th response, just before the test stimulus). A similar result was obtained when we examined the effect of repetitive stimulation of the $\mathrm{PW}$ on the response to $\mathrm{AW}$, i.e., the $\mathrm{AW}$ test response was unaffected by PW adaptation (Fig. 6D). Thus, for this layer 4 cell, repetitive stimulation of either the $\mathrm{PW}$ or the AW had a negligible effect on its response to the other whisker. The two additional examples shown in Figure 7, one of a layer 4 cell (Fig. $7 A-D$ ) and the other of a layer $2 / 3$ cell (Fig. $7 E-H$ ), demonstrate again that response adaptation by means of repetitive stimulation of one whisker has only a minor effect on the response to subsequent stimulation of the neighboring whisker.

Population data for cross-whisker adaptation are presented in Figure 8. For each cell, we measured the peak amplitude of the control response (represented by the coordinate of the data point on the abscissa of each panel), the adapted response (the 20th "adapted response"; filled circles), and the test response (open
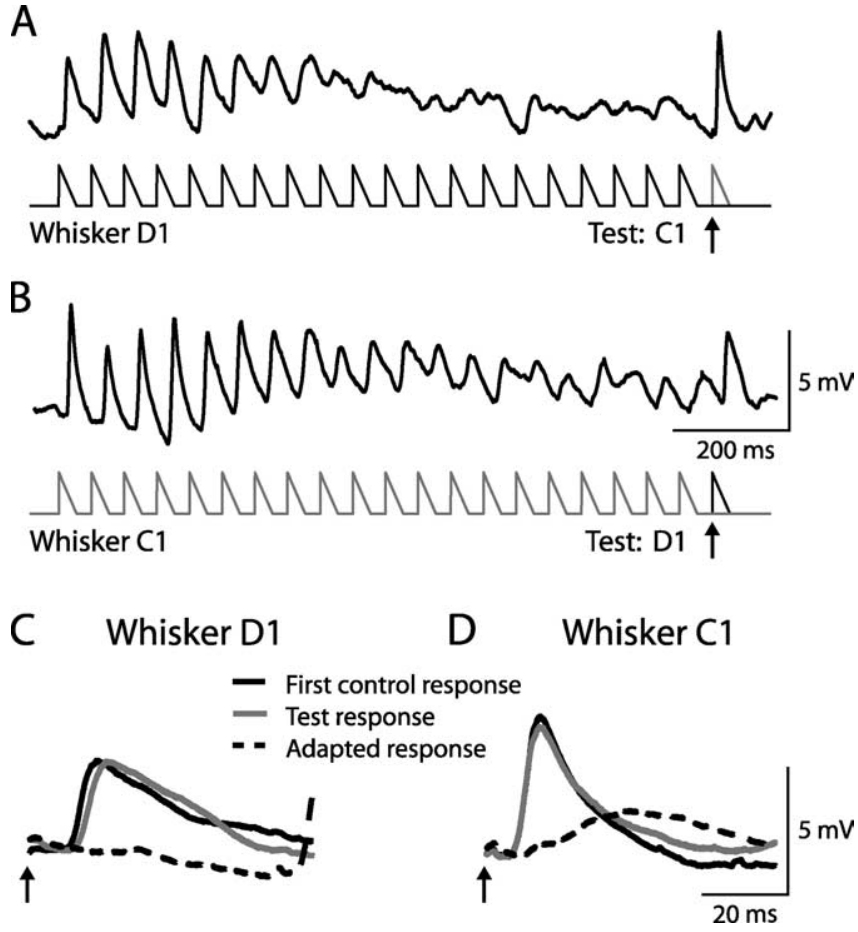

Figure 6. Cross-whisker adaptation of a layer 4 cell. $\boldsymbol{A}$, Average subthreshold response to repetitive stimulation of the best AW ( 20 stimuli at $18 \mathrm{~Hz})$, followed by a single stimulation of the PW (arrow). The stimulation waveform is presented below the average membrane potential response. $\boldsymbol{B}$, Reciprocally, the average response to repetitive stimulation of the $P W$ followed by a single stimulation of the best AW. C, D, Overlay of the control response (measured from the first average response of the reciprocal protocol), the test response (measured from the response to the single deflection after repetitive stimulation of the other whisker), and the adapted response (measured from the last response before the reciprocal test) shows that preadapting this cell by stimulating one whisker has no effect on its subsequent response to a neighboring whisker (stimulus onset is depicted by an arrow).

circles). These values are shown for the PW and AW of layer 4 ( $A$ and $B$, respectively) and of layer $2 / 3$ ( $C$ and $D$, respectively). In these plots, the PW was selected on the basis of at least two of the three criteria [shorter latency to response, shorter rise time, and larger RI (Fig. 3)]. In layer 4 cells, the 20th average response to deflection of the PW (amplitude of $2.35 \pm 0.35 \mathrm{mV} ; n=13$ ) or the AW $(0.80 \pm 0.24 \mathrm{mV})$ response showed significant adaptation $(p<0.001)$ compared with their corresponding control responses $(7.71 \pm 0.87$ and $4.59 \pm 0.67 \mathrm{mV}$, respectively). Nevertheless, the PW and AW test responses (7.79 \pm 0.82 and $4.20 \pm$ $0.79 \mathrm{mV}$, respectively) did not differ significantly from their controls ( $p=0.82$ and $p=0.26$, respectively). Slightly larger reductions were observed in the test responses of layer $2 / 3$ cells. Even in these cells, however, relative to their respective controls, the PW test response $(n=18)$ was only $8 \%$ smaller $(6.09 \pm 1.06 \mathrm{mV}$ compared with $6.64 \pm 0.87 \mathrm{mV} ; p=0.29)$, and the test response of the AW was $16 \%$ smaller $(4.60 \pm 0.42 \mathrm{mV}$ compared with $5.49 \pm 0.48 \mathrm{mV} ; p=0.016)$. These data demonstrate that adaptation is highly specific to the stimulated whisker.

\section{Discussion}

The data in this study indicate that, in the rat barrel cortex, despite the large receptive field size of the neurons, adaptation of the synaptic response is highly specific to the stimulated whisker. The marked input specificity of adaptation in the cortex implies that adaptation is not induced by intrinsic mechanisms or buildup of inhibition. These results and the virtual absence of gradual adap- 
A

A

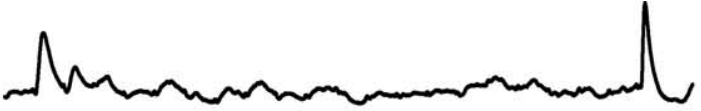

MNMNNMNNMNMNM Whisker D3

Test:D2 $\uparrow$

B

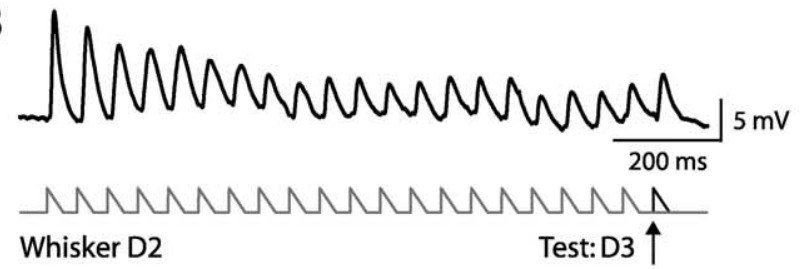

C Whisker D3

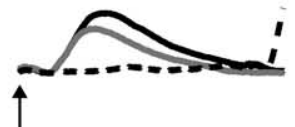

D Whisker D2

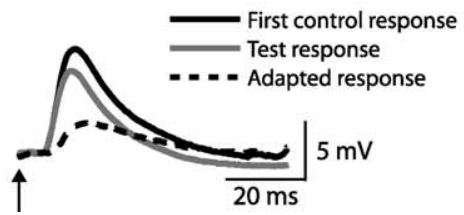

E

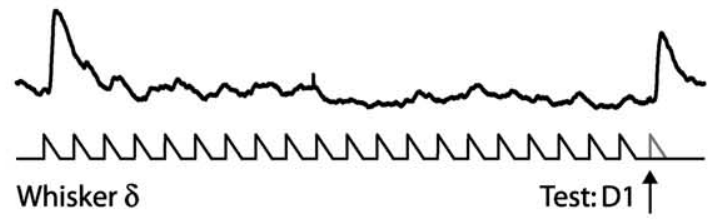

$\mathrm{F}$

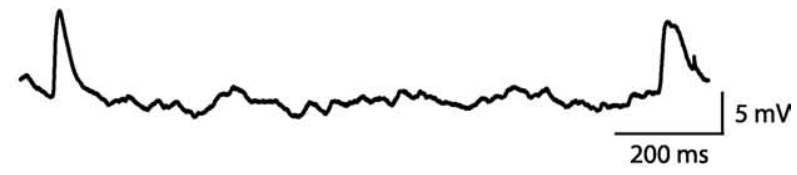

MNMNANMNMNMNMNA Whisker D1

Test: $\delta \uparrow$
G

Whisker $\delta$

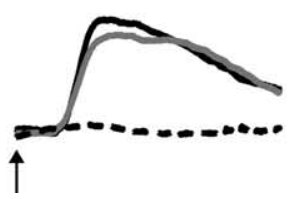

H Whisker D1

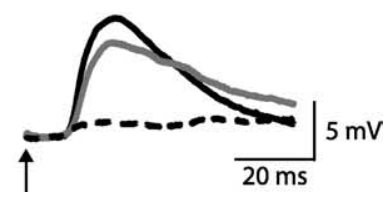

Figure 7. Cross-whisker adaptation for two additional cells. A--D, A layer 4 cell shows only moderate reduction in the test response compared with its control. $\boldsymbol{E}-\boldsymbol{H}$, Minor cross-whisker adaptation effect in a layer $2 / 3$ cell. Format of representation is similar to that in Figure 6.
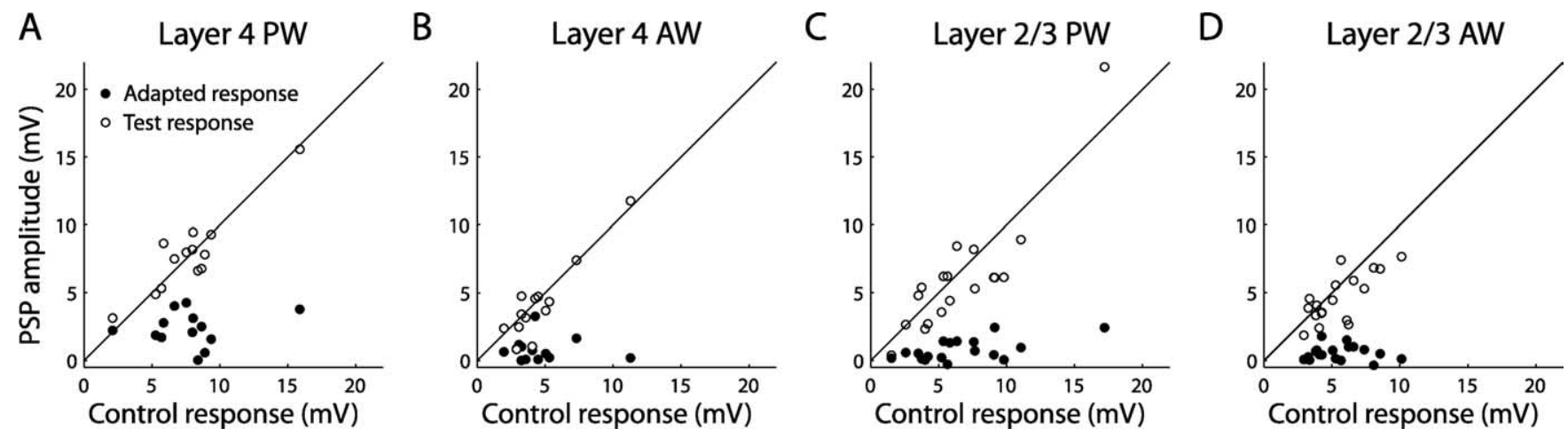

Figure 8. Cross-whisker adaptation of layer 4 and layer $2 / 3$ cells. Adaptation induced by repetitive stimulation of one whisker (the PW or the best AW) had only a minor effect on the response to subsequent deflection of the neighboring whisker. Control response for each of the two whiskers is presented in the abscissa of each panel. The amplitude of the adapted response (the 20 th response to identical stimuli; see Figs. 6 and 7 ) is plotted against these values (filled circles), together with the test response amplitude (open circles) measured from single subsequent stimulation. In all cases (different layers and whiskers), on average, the adapted response was significantly smaller than the corresponding control response $(p<0.001)$. In general, the test response for all layers was similar in amplitude to the corresponding control response; however, a small but significant reduction was measured only for AW stimulation after repetitive stimulation of the PW in layer $2 / 3$ cells $(p=0.016)$.

tation in the thalamus suggest that the major process responsible for adaptation occurs at a point at which the pathways that convey inputs from different whiskers are separate, probably at the thalamocortical synapse. Thus, we suggest that, under our experimental conditions, the AW response of layer 4 cells was generated not by the same thalamic cells that convey the PW response but rather by integration at the cortex or by convergence of multiple thalamic inputs.

Thalamic cells showed almost no gradual adaptation (Fig. 2), consistent with other studies of VPM cells using extracellular technique (Hellweg et al., 1977; Sosnik et al., 2001; Hartings et al., 2003; Khatri et al., 2004). As in other studies, cortical cells, including layer 4 cells, adapted considerably more than thalamic cells, mostly at frequencies above $10 \mathrm{~Hz}$ (Ahissar et al., 2001; Garabedian et al., 2003; Khatri et al., 2004), and supragranular neurons adapted more than layer 4 cells (Ahissar et al., 2000, 2001).
Several mechanisms could account for cortical adaptation. (1) Short-term synaptic depression of thalamocortical synapses contributes to the reduction in synaptic response during adaptation (Gil et al., 1999; Castro-Alamancos, 2002; Chung et al., 2002; Beierlein et al., 2003). However, recent studies have shown that, during arousal states, adaptation is profoundly reduced because the thalamocortical circuits are already suppressed (CastroAlamancos, 2002, 2004). (2) Synaptic depression of cortical synapses (Petersen, 2002; Beierlein et al., 2003; Cowan and Stricker, 2004) can lead to reduction in intracortical amplification in layer 4 (Beierlein et al., 2002). (3) Buildup of cortical inhibition during adaptation (Dealy and Tolhurst, 1974) can shunt the response to sensory inputs. (4) Intrinsic mechanisms, such as the prolonged hyperpolarization described for cortical cells in the visual cortex (Carandini and Ferster, 1997; Sanchez-Vives et al., 2000), may enhance adaptation. The strong specificity of adaptation to the stimulated whisker implies, however, that neither intrinsic mech- 
anisms nor buildup of inhibition are likely to be involved in adaptation to whisker stimulation. If these two mechanisms were involved, we would expect to find a large reduction in the test response of one whisker as a consequence of adaptation induced by stimulation of a neighboring whisker. Thus, the above considerations suggest that the underlying mechanism of adaptation probably involves short-term synaptic depression of thalamocortical synapses of thalamic cells or adaptation of the cortical circuit by non-intrinsic mechanisms.

Our study sheds light on the synaptic origin of the AW response in the barrel cortex. Three main mechanisms have been suggested to account for the AW response in layer 4. (1) The cortical multiwhisker response might reflect the receptive field of thalamic cells. (2) The response of AW might arise via intracortical connections. (3) Diverging inputs from different thalamic barreloids might converge on a single cortical cell.

The first mechanism is supported by lesion of the trigeminal nucleus interpolaris, which led to a reduction in size of the receptive fields of thalamic (Rhoades et al., 1987; Friedberg et al., 2004; Timofeeva et al., 2004; Kwegyir-Afful et al., 2005) and cortical (Kwegyir-Afful et al., 2005) neurons. Those studies, together with spike cross-correlograms of thalamocortical pairs of neurons (Bruno and Simons, 2002), suggested that the cortical AW and PW responses are driven by the same thalamic cells. Our adaptation experiments, together with the small size of the VPM receptive field (Fig. 2), suggest that, at least under our experimental conditions, no significant part of the AW synaptic response was likely to be transmitted by the same thalamic fibers as those responsible for the PW response. If that was indeed the case, as a result of short-term synaptic depression of thalamocortical synapses (Chung et al., 2002), the AW test response would be significantly reduced. There are several possible explanations for the discrepancy between our data and studies demonstrating large receptive field of thalamic VPM cells. Under light anesthesia, the receptive fields of thalamic VPM cells are larger than under deep anesthesia (Friedberg et al., 1999). Anesthesia in our experiments was at approximately level III-3 to III-2 (see Materials and Methods), supported by experiments in which we mapped the receptive field sizes of thalamic VPM cells while recording EEG activity. Thus, the small sizes of the receptive fields of VPM cells in our study are not a result of deep anesthesia. Our recorded VPM cells fired at similar rates to that reported in two extracellular studies, in which whisker stimulation was similar to that applied in our study (Hartings et al., 2003; Khatri et al., 2004). It thus seems that intracellular recordings do not alter the ability of thalamic cells to fire at the physiological rate, validating their small receptive field size. It is possible that the age of the rats (which were younger in our study than in the two studies cited above) plays a role in determining the receptive field size in the thalamus. This possibility is supported by similar findings of another intracellular study of VPM cells in young rats (Brecht and Sakmann, 2002a).

According to the second mechanism, the AW response is generated by cortical inputs from adjacent barrels (Armstrong-James and Callahan, 1991), supported by pharmacological inactivation of adjacent cortical barrels (Fox et al., 2003) and by activation of adjacent barrels in brain slices (Schubert et al., 2003). Furthermore, reconstructions of axonal projections of in vivo intracellularly recorded cells (Brecht and Sakmann, 2002b) suggest that the AW response might originate in intracortical pathways. That the cortical response to the AW was found here to be barely affected by adaptation of the PW implies that only a small fraction of the circuit in layer 4 undergoes adaptation via activation of its neigh- boring barrel, in agreement with the notion that barrels are functionally independent (Petersen and Sakmann, 2001).

The third possible source of the AW response is that diverging inputs from different thalamic cells, each responding primarily to a different whisker, synapse on the same cortical cell. This is partially supported by an anatomical study demonstrating that a small fraction of axonal collateral of thalamocortical VPM cells terminate in other barrels outside the primary barrel (Arnold et al., 2001) and was considered in another study of the barrel cortex (Brecht and Sakmann, 2002b). A recent study, however, failed to provide evidence for direct connections between a barreloid and an unaligned cortical barrel (Bruno and Sakmann, 2006). If diverging thalamic inputs are responsible for the AW response and unless the kinetics of short-term synaptic depression of the AW thalamocortical synapse differ from the kinetics for the PW inputs, we would expect, in contrast to our findings (Figs. 3, 4) and those of a recent extracellular study (Ego-Stengel et al., 2005), that the rates of adaptation to PW and AW stimulation would be similar. An additional synapse along the pathway and nonlinearity of the spike threshold would speed up adaptation at higher stages (Boudreau and Ferster, 2005). Thus, the difference between PW adaptation and AW adaptation might reflect the existence of an additional synapse along the pathway of the AW, which however is inconsistent with the hypothesis of diverging thalamic inputs.

In summary, we hypothesize that a cortical pathway between adjacent barrels is responsible for the AW response. However, the response in each barrel is independent of previous adaptation of adjacent barrels, suggesting that adaptation occurs at the thalamocortical synapse. Additional studies are needed to acquire direct evidence for the role of direct cortical inputs in shaping the response to adjacent whiskers.

Because layer 4 cells adapt during stimulation, it is more difficult to interpret the data obtained by our cross-whisker experiments for layer $2 / 3$ cells. Ascending inputs from layer 4 to layer 2/3 (Bender et al., 2003; Shepherd and Svoboda, 2005) are likely to convey integrated inputs from the PW and from AWs and might undergo additional adaptation (Beck et al., 2005), resulting in a reduction in the test response. However, because of firing suppression of layer 4 cells caused by adaptation, ascending inputs may be able to transmit the subsequent response to adjacent whisker stimulation. Our data cannot exclude the possibility that AW response of layer $2 / 3$ cells can also arrive from adjacent column (Reyes and Sakmann, 1999; Petersen et al., 2003; Derdikman et al., 2006). These possibilities may explain the small but significant reduction of the AW test response of layer $2 / 3$ cells (Fig. 8). Thus, compared with layer 4 , fewer conclusions can be drawn regarding whisker integration at layer $2 / 3$ cells. Nevertheless, adaptation in supragranular cells is also highly specific, suggesting that it is not induced by intrinsic or by inhibitory mechanisms.

The implications of whisker-specific adaptation on the behavior of the rat are unclear. A sensation of natural texture during whisking causes high-frequency velocity vibrations of the whisker (Arabzadeh et al., 2003, 2005), possibly leading to substantial adaptation at the cortex (Khatri et al., 2004). We suggest that adaptation might not reduce the response of a neighboring whisker to subsequent contact with the texture. Moreover, despite the traditional view that movements of multiple whiskers during natural whisking are highly synchronized, recent fast video imaging studies show that neighboring whiskers can move independently during whisking (Sachdev et al., 2002; Knutsen et al., 2006). Thus, during natural behavior, each whisker might be stimulated inde- 
pendently. On the basis of the present finding that adaptation is highly specific, we suggest that neurons at the cortex encode inputs from different whiskers, regardless of previous adaptation caused by stimulation of other whiskers. Specific whisker adaptation might therefore optimize information throughput during object recognition.

\section{References}

Adorjan P, Piepenbrock C, Obermayer K (1999) Contrast adaptation and infomax in visual cortical neurons. Rev Neurosci 10:181-200.

Ahissar E, Sosnik R, Haidarliu S (2000) Transformation from temporal to rate coding in a somatosensory thalamocortical pathway. Nature 406:302-306.

Ahissar E, Sosnik R, Bagdasarian K, Haidarliu S (2001) Temporal frequency of whisker movement. II. Laminar organization of cortical representations. J Neurophysiol 86:354-367.

Arabzadeh E, Petersen RS, Diamond ME (2003) Encoding of whisker vibration by rat barrel cortex neurons: implications for texture discrimination. J Neurosci 23:9146-9154.

Arabzadeh E, Zorzin E, Diamond ME (2005) Neuronal encoding of texture in the whisker sensory pathway. PLoS Biol 3:e17.

Armstrong-James M, Callahan CA (1991) Thalamo-cortical processing of vibrissal information in the rat. II. Spatiotemporal convergence in the thalamic ventroposterior medial nucleus (VPm) and its relevance to generation of receptive fields of S1 cortical "barrel" neurones. J Comp Neurol 303:211-224.

Arnold PB, Li CX, Waters RS (2001) Thalamocortical arbors extend beyond single cortical barrels: an in vivo intracellular tracing study in rat. Exp Brain Res 136:152-168.

Beck O, Chistiakova M, Obermayer K, Volgushev M (2005) Adaptation at synaptic connections to layer $2 / 3$ pyramidal cells in rat visual cortex. J Neurophysiol 94:363-376.

Beierlein M, Fall CP, Rinzel J, Yuste R (2002) Thalamocortical bursts trigger recurrent activity in neocortical networks: layer 4 as a frequencydependent gate. J Neurosci 22:9885-9894.

Beierlein M, Gibson JR, Connors BW (2003) Two dynamically distinct inhibitory networks in layer 4 of the neocortex. J Neurophysiol 90:2987-3000.

Bender KJ, Rangel J, Feldman DE (2003) Development of columnar topography in the excitatory layer 4 to layer $2 / 3$ projection in rat barrel cortex. J Neurosci 23:8759-8770.

Berg RW, Kleinfeld D (2003) Rhythmic whisking by rat: retraction as well as protraction of the vibrissae is under active muscular control. J Neurophysiol 89:104-117.

Boudreau CE, Ferster D (2005) Short-term depression in thalamocortical synapses of cat primary visual cortex. J Neurosci 25:7179-7190.

Brecht M, Sakmann B (2002a) Whisker maps of neuronal subclasses of the rat ventral posterior medial thalamus, identified by whole-cell voltage recording and morphological reconstruction. J Physiol (Lond) 538:495-515.

Brecht M, Sakmann B (2002b) Dynamic representation of whisker deflection by synaptic potentials in spiny stellate and pyramidal cells in the barrels and septa of layer 4 rat somatosensory cortex. J Physiol (Lond) 543:49-70.

Brecht M, Roth A, Sakmann B (2003) Dynamic receptive fields of reconstructed pyramidal cells in layers 3 and 2 of rat somatosensory barrel cortex. J Physiol (Lond) 553:243-265.

Bruno RM, Sakmann B (2006) Cortex is driven by weak but synchronously active thalamocortical synapses. Science 312:1622-1627.

Bruno RM, Simons DJ (2002) Feedforward mechanisms of excitatory and inhibitory cortical receptive fields. J Neurosci 22:10966-10975.

Carandini M, Ferster D (1997) A tonic hyperpolarization underlying contrast adaptation in cat visual cortex. Science 276:949-952.

Castro-Alamancos MA (2002) Role of thalamocortical sensory suppression during arousal: focusing sensory inputs in neocortex. J Neurosci 22:9651-9655.

Castro-Alamancos MA (2004) Absence of rapid sensory adaptation in neocortex during information processing states. Neuron 41:455-464.

Chung S, Ferster D (1998) Strength and orientation tuning of the thalamic input to simple cells revealed by electrically evoked cortical suppression. Neuron 20:1177-1189.

Chung S, Li X, Nelson SB (2002) Short-term depression at thalamocortical synapses contributes to rapid adaptation of cortical sensory responses in vivo. Neuron 34:437-446.

Condon CD, Weinberger NM (1991) Habituation produces frequencyspecific plasticity of receptive fields in the auditory cortex. Behav Neurosci 105:416-430.

Connors BW, Prince DA (1982) Effects of local anesthetic QX-314 on the membrane properties of hippocampal pyramidal neurons. J Pharmacol Exp Ther 220:476-481.

Cowan AI, Stricker C (2004) Functional connectivity in layer IV local excitatory circuits of rat somatosensory cortex. J Neurophysiol 92:2137-2150.

Dealy RS, Tolhurst DJ (1974) Is spatial adaptation an after-effect of prolonged inhibition? J Physiol (Lond) 241:261-270.

Derdikman D, Yu C, Haidarliu S, Bagdasarian K, Arieli A, Ahissar E (2006) Layer-specific touch-dependent facilitation and depression in the somatosensory cortex during active whisking. J Neurosci 26:9538-9547.

Dragoi V, Sharma J, Sur M (2000) Adaptation-induced plasticity of orientation tuning in adult visual cortex. Neuron 28:287-298.

Ego-Stengel V, Mello e Souza T, Jacob V, Shulz DE (2005) Spatiotemporal characteristics of neuronal sensory integration in the barrel cortex of the rat. J Neurophysiol 93:1450-1467.

Fox K, Wright N, Wallace H, Glazewski S (2003) The origin of cortical surround receptive fields studied in the barrel cortex. J Neurosci 23:8380-8391.

Friedberg MH, Lee SM, Ebner FF (1999) Modulation of receptive field properties of thalamic somatosensory neurons by the depth of anesthesia. J Neurophysiol 81:2243-2252.

Friedberg MH, Lee SM, Ebner FF (2004) The contribution of the principal and spinal trigeminal nuclei to the receptive field properties of thalamic VPM neurons in the rat. J Neurocytol 33:75-85.

Gabernet L, Jadhav SP, Feldman DE, Carandini M, Scanziani M (2005) Somatosensory integration controlled by dynamic thalamocortical feedforward inhibition. Neuron 48:315-327.

Garabedian CE, Jones SR, Merzenich MM, Dale A, Moore CI (2003) Bandpass response properties of rat SI neurons. J Neurophysiol 90:1379-1391.

Ghazanfar AA, Nicolelis MA (1999) Spatiotemporal properties of layer V neurons of the rat primary somatosensory cortex. Cereb Cortex 9:348-361.

Gil Z, Connors BW, Amitai Y (1999) Efficacy of thalamocortical and intracortical synaptic connections: quanta, innervation, and reliability. Neuron 23:385-397.

Goldreich D, Kyriazi HT, Simons DJ (1999) Functional independence of layer IV barrels in rodent somatosensory cortex. J Neurophysiol 82:1311-1316.

Hartings JA, Temereanca S, Simons DJ (2003) Processing of periodic whisker deflections by neurons in the ventroposterior medial and thalamic reticular nuclei. J Neurophysiol 90:3087-3094.

Hellweg FC, Schultz W, Creutzfeldt OD (1977) Extracellular and intracellular recordings from cat's cortical whisker projection area: thalamocortical response transformation. J Neurophysiol 40:463-479.

Higley MJ, Contreras D (2003) Nonlinear integration of sensory responses in the rat barrel cortex: an intracellular study in vivo. J Neurosci 23:10190-10200.

Higley MJ, Contreras D (2005) Integration of synaptic responses to neighboring whiskers in rat barrel cortex in vivo. J Neurophysiol 93:1920-1934.

Horikawa K, Armstrong WE (1988) A versatile means of intracellular labeling: injection of biocytin and its detection with avidin conjugates. J Neurosci Methods 25:1-11.

Khatri V, Hartings JA, Simons DJ (2004) Adaptation in thalamic barreloid and cortical barrel neurons to periodic whisker deflections varying in frequency and velocity. J Neurophysiol 92:3244-3254.

Knutsen PM, Pietr M, Ahissar E (2006) Haptic object localization in the vibrissal system: behavior and performance. J Neurosci 26:8451-8464.

Kwegyir-Afful EE, Bruno RM, Simons DJ, Keller A (2005) The role of thalamic inputs in surround receptive fields of barrel neurons. J Neurosci 25:5926-5934.

Lampl I, Reichova I, Ferster D (1999) Synchronous membrane potential fluctuations in neurons of the cat visual cortex. Neuron 22:361-374.

Land PW, Simons DJ (1985) Cytochrome oxidase staining in the rat SmI barrel cortex. J Comp Neurol 238:225-235.

Malone BJ, Scott BH, Semple MN (2002) Context-dependent adaptive cod- 
ing of interaural phase disparity in the auditory cortex of awake macaques. J Neurosci 22:4625-4638.

Manns ID, Sakmann B, Brecht M (2004) Sub- and suprathreshold receptive field properties of pyramidal neurones in layers $5 \mathrm{~A}$ and $5 \mathrm{~B}$ of rat somatosensory barrel cortex. J Physiol (Lond) 556:601-622.

Moore CI, Nelson SB (1998) Spatio-temporal subthreshold receptive fields in the vibrissa representation of rat primary somatosensory cortex. J Neurophysiol 80:2882-2892.

Movshon JA, Lennie P (1979) Pattern-selective adaptation in visual cortical neurones. Nature 278:850-852.

Muller JR, Metha AB, Krauskopf J, Lennie P (1999) Rapid adaptation in visual cortex to the structure of images. Science 285:1405-1408.

Nathan T, Jensen MS, Lambert JD (1990) The slow inhibitory postsynaptic potential in rat hippocampal CA1 neurones is blocked by intracellular injection of QX-314. Neurosci Lett 110:309-313.

Perkins KL, Wong RK (1995) Intracellular QX-314 blocks the hyperpolarization-activated inward current Iq in hippocampal CA1 pyramidal cells. J Neurophysiol 73:911-915.

Petersen CC (2002) Short-term dynamics of synaptic transmission within the excitatory neuronal network of rat layer 4 barrel cortex. J Neurophysiol 87:2904-2914.

Petersen CC, Sakmann B (2001) Functionally independent columns of rat somatosensory barrel cortex revealed with voltage-sensitive dye imaging. J Neurosci 21:8435-8446.

Petersen CC, Grinvald A, Sakmann B (2003) Spatiotemporal dynamics of sensory responses in layer $2 / 3$ of rat barrel cortex measured in vivo by voltage-sensitive dye imaging combined with whole-cell voltage recordings and neuron reconstructions. J Neurosci 23:1298-1309.

Priebe NJ, Ferster D (2005) Direction selectivity of excitation and inhibition in simple cells of the cat primary visual cortex. Neuron 45:133-145.

Reyes A, Sakmann B (1999) Developmental switch in the short-term modification of unitary EPSPs evoked in layer 2/3 and layer 5 pyramidal neurons of rat neocortex. J Neurosci 19:3827-3835.

Rhoades RW, Belford GR, Killackey HP (1987) Receptive-field properties of rat ventral posterior medial neurons before and after selective kainic acid lesions of the trigeminal brain stem complex. J Neurophysiol 57:1577-1600.

Sachdev RN, Sato T, Ebner FF (2002) Divergent movement of adjacent whiskers. J Neurophysiol 87:1440-1448.

Sanchez-Vives MV, Nowak LG, McCormick DA (2000) Cellular mechanisms of long-lasting adaptation in visual cortical neurons in vitro. J Neurosci 20:4286-4299.

Saul AB, Cynader MS (1989) Adaptation in single units in visual cortex: the tuning of aftereffects in the spatial domain. Vis Neurosci 2:593-607.

Schubert D, Kotter R, Zilles K, Luhmann HJ, Staiger JF (2003) Cell typespecific circuits of cortical layer IV spiny neurons. J Neurosci 23:2961-2970.

Shepherd GM, Svoboda K (2005) Laminar and columnar organization of ascending excitatory projections to layer $2 / 3$ pyramidal neurons in rat barrel cortex. J Neurosci 25:5670-5679.

Simons DJ (1985) Temporal and spatial integration in the rat SI vibrissa cortex. J Neurophysiol 54:615-635.

Simons DJ, Carvell GE (1989) Thalamocortical response transformation in the rat vibrissa/barrel system. J Neurophysiol 61:311-330.

Sosnik R, Haidarliu S, Ahissar E (2001) Temporal frequency of whisker movement. I. Representations in brain stem and thalamus. J Neurophysiol 86:339-353.

Timofeeva E, Lavallee P, Arsenault D, Deschenes M (2004) Synthesis of multiwhisker-receptive fields in subcortical stations of the vibrissa system. J Neurophysiol 91:1510-1515.

Ulanovsky N, Las L, Nelken I (2003) Processing of low-probability sounds by cortical neurons. Nat Neurosci 6:391-398.

Wilent WB, Contreras D (2005) Dynamics of excitation and inhibition underlying stimulus selectivity in rat somatosensory cortex. Nat Neurosci 8:1364-1370.

Woolsey TA, Van der Loos H (1970) The structural organization of layer IV in the somatosensory region (SI) of mouse cerebral cortex. The description of a cortical field composed of discrete cytoarchitectonic units. Brain Res 17:205-242. 